\title{
SCALED CONJUGATE GRADIENT BASED DECISION SUPPORT SYSTEM FOR AUTOMATED DIAGNOSIS OF SKIN CANCER
}

\author{
Ammara Masood, Adel Al-Jumaily, Yee Mon Aung \\ University of Technology Sydney, Broadway 2007, Australia \\ ammara.masood@student.uts.edu.au, Adel.Al-Jumaily@uts.edu.au, Yee.M.Aung@student.uts.edu.au
}

\begin{abstract}
Melanoma is the most deathful form of skin cancer but early diagnosis can ensure a high rate of survival. Early diagnosis is one of the greatest challenges due to lack of experience of general practitioners (GPs). This paper presents a clinical decision support system designed for the use of general practitioners, aiming to save time and resources in the diagnostic process. Segmentation, pattern recognition, and lesion detection are the important steps in the proposed decision support system. The system analyses the images to extract the affected area using a novel proposed segmentation method. It determinates the underlying features which indicate the difference between melanoma and benign images and makes a decision. Considering the efficiency of neural networks in classification of complex data, scaled conjugate gradient based neural network is used for classification. The presented work also considers analyzed performance of other efficient neural network training algorithms on the specific skin lesion diagnostic problem and discussed the corresponding findings. The best diagnostic rates obtained through the proposed decision support system are around $92 \%$.
\end{abstract}

\section{KEY WORDS}

Neural network, Diagnostic systems, Segmentation, Feature extraction, Classification.

\section{Introduction}

Malignant melanoma is one of the leading causes of death, and a rapid increase in the number of cases of melanoma is observed in Europe, North America, and Australia over the last decade [1]. An estimated 76,250 new cases of invasive melanoma were diagnosed in the US in 2012, with an estimated 9,180 resulting in death [2]. Over 1,890 Australians die from skin cancer each year [3]. From treatment point of view, skin cancer is one of the most expensive forms of cancer [4]. In 2001, it was estimated the treatment of non-melanoma skin cancer cost \$264 million and melanoma \$30 million. But early diagnosis can make the situation better as melanoma has near $95 \%$ cure rate if diagnosed and treated in early stages [4]. In visual diagnosing, it is hard for medical professionals to differentiate normal from abnormal mole, and general practitioners (GP) do not have core expertise to diagnose skin cancers. Dermatologist's data indicate that even in specialized centers diagnostic accuracy is only about $60 \%$ [5], and at the same time they are overloaded by GP referrals.

This study proposes an automated system for discrimination between melanocytic nevi and malignant melanoma. The benefits of computerized diagnostic tools are twofold: (1) to be used as a standalone warning tools for helping the GPs in early diagnosis; (2) to provide quantitative information about the lesion for experts to be considered during biopsy decision making.

The approach of developing a Computer Aided Diagnostic (CAD) system for diagnosis of skin cancer is to find the location of a lesion and also to determine an estimate of the probability of a disease. Differentiation of malignant melanoma images demands efficient image processing, segmentation and feature extraction and classification algorithms. There are various diagnostic systems present in literature [6-9] but as discussed in [10] detailed research is necessary to make the best choice and setting of the benchmarks for diagnostic system development and validation. This paper presents a part of our research being carried out to come up with a best combination of segmentation, feature extraction and classification algorithms which can consequently form a basis of more generalized and accurate skin cancer diagnostic system. The diagnostic model used in this paper is shown in Fig. 1.
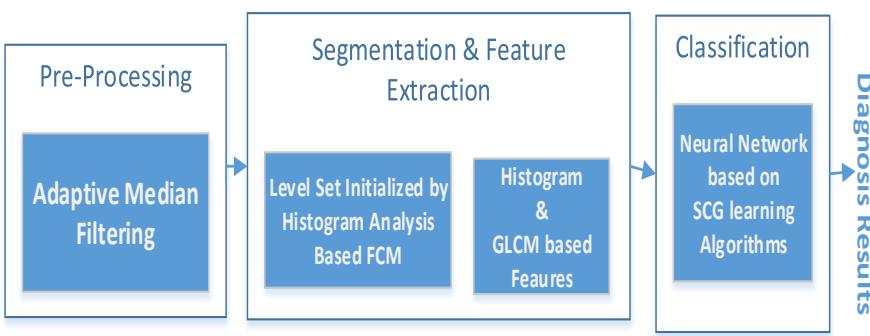

Figure. 1 Computer Aided Diagnostic Support System

The decision support system proposed in this paper uses adaptive median filter for pre-processing of image to reduce the ill effects and various artifacts like hair that may be present in the dermoscopic images. It is followed by the detection of the lesion by image segmentation technique. Histogram based fuzzy $\mathrm{C}$ means thresholding algorithm presented in [11] is being used in these experiments. This algorithm provided efficient 
segmentation results as compared to other segmentation results used in literature, the comparative analysis is presented in [12]. Once the lesion is localized, different intensity and texture based features is quantified using gray level co-occurrence matrix. Finally, artificial neural network based on scaled conjugate gradient algorithm is used for classification of cancerous and non-cancerous skin lesions. In order to justify the performance of the proposed method, classification results of the proposed method are also compared with the diagnosis results of neural networks based on other popular learning algorithms.

This paper is organized as follows: Section 2 describes the computer-aided diagnosing (CAD) which consists of the pre-processing, segmentation, the features extraction and classification. Section3 presents the experimental results, comparative analysis and Discussion, and finally section for conclusions and intended future work.

\section{Material and Methods}

\subsection{Experimental Dataset}

A clinical database of dermoscopic and clinical view lesion images were obtained from different sources but most of the images came from the Sydney Melanoma Diagnostic Centre, Royal Prince Alfred Hospital, Vienna General Hospital and Hôpital Charles Nicolle CHU Rouen, France. The images have been stored in the RGB color format having different dimensions which were rescaled to a resolution of $150 \times 150$ with bit depth 24 and size around $68 \mathrm{~KB}$. The database was further subdivided into 2 categories (1) benign melanocytic lesions and (2) malignant melanoma lesions. Since there was no control over the image acquisition system and camera calibration and settings, images that had at least one of the following problems were not used in the study: (i) the lesion that does not fit completely in the image frame and (ii) insufficient contrast between the lesion and the background skin or (iii) if the images has a shadow that is hard to remove even after pre-processing. This selectivity was necessary to ensure accurate segmentation and reliable feature extraction. This selectivity in data set is practically justified in the actual use of the method, because the problems like taking care of proper image frame, avoiding the shadow and improper illumination can be easily rectified by the high quality image acquisition devices available these days. Fig. 2 shows sample images that were eliminated using these criteria. A total of 135 images ( 81 benign and 54 melanoma) that were free from the above mentioned problems were included in the experimental data set.
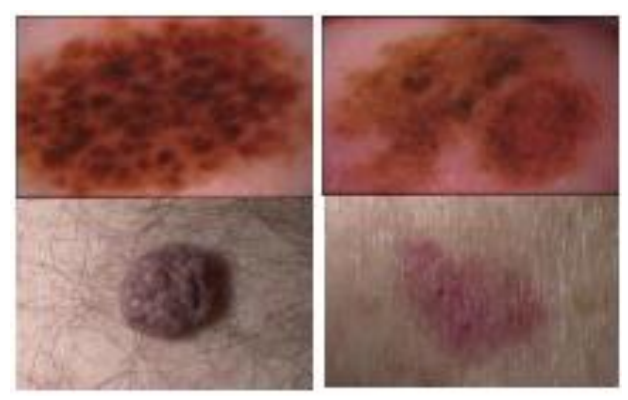

Figure. 2 Types of Skin Images not Included in Experiments

\subsection{Pre-Processing}

For there are certain extraneous artifacts such as skin texture, air bubbles, dermoscopic gel, presence of ruler markings and hair that make border detection a bit difficult. In order to reduce the effect of these artifacts on segmentation results, it is necessary to pre-process the images with a smoothing filter. We found, by experiments, that the best segmentation results were obtained using adaptive median filter[13]. The median filter also performs well as long as the spatial density of the impulse noise is not too large. However the adaptive median filtering has a better capability to handle impulse noise with even larger probabilities. An additional benefit of the adaptive median filter is that it seeks to preserve details while smoothing the non-impulse noise[14]. Considering the high level of noise that may be present in skin lesion images and the need of preserving structural details, the adaptive median algorithm performed quite well.

\subsection{Segmentation}

Segmentation algorithm, histogram analysis based fuzzy C mean algorithm for Level Set initialization (H-FCMLS) used in this work is presented in Fig. 3. It is found that the practical significance of fuzzy clustering for multidimensional feature space is of great importance when it comes to skin cancer diagnosis. In this case deterministic misclassification can be very costly and a sophisticated thresholding procedure is required for increasing the accuracy of segmentation results. In the proposed algorithm, histogram analysis of image was done to see average intensity distribution in the images and then the hard threshold was selected between classes with dominant intensity values. Thus, this method solves the difficulty that may arise in finding effective threshold automatically for different images regardless of intensity variety. This method was further used as an initializing step for complex segmentation methods having spatial information. 


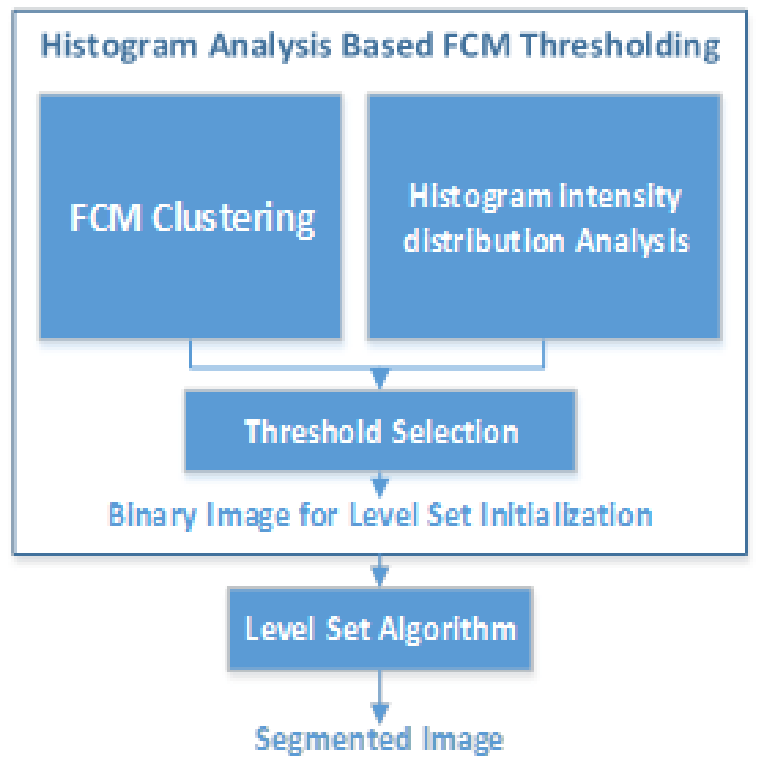

Figure. 3 Block Diagram of Segmentation Algorithm (H-FCM-LS)

Histogram analysis based fuzzy $\mathrm{C}$ mean algorithm is shown in Fig. 4. The mathematical details of histogram analysis based fuzzy $\mathrm{C}$ mean algorithm can be found in [15] while the details of overall segmentation algorithm can be found in [16]. The results of the segmentation method (histogram analysis based fuzzy $\mathrm{C}$ mean algorithm for Level Set initialization (H-FCM- LS)) are compared with other well-known segmentation methods like adaptive thresholding, fuzzy $\mathrm{C}$ mean clustering, $\mathrm{K}$ mean clustering and other thresholding and segmentation methods and it is shown in $[11,12]$ that this methods gives more accurate segmentation results for skin lesion images.

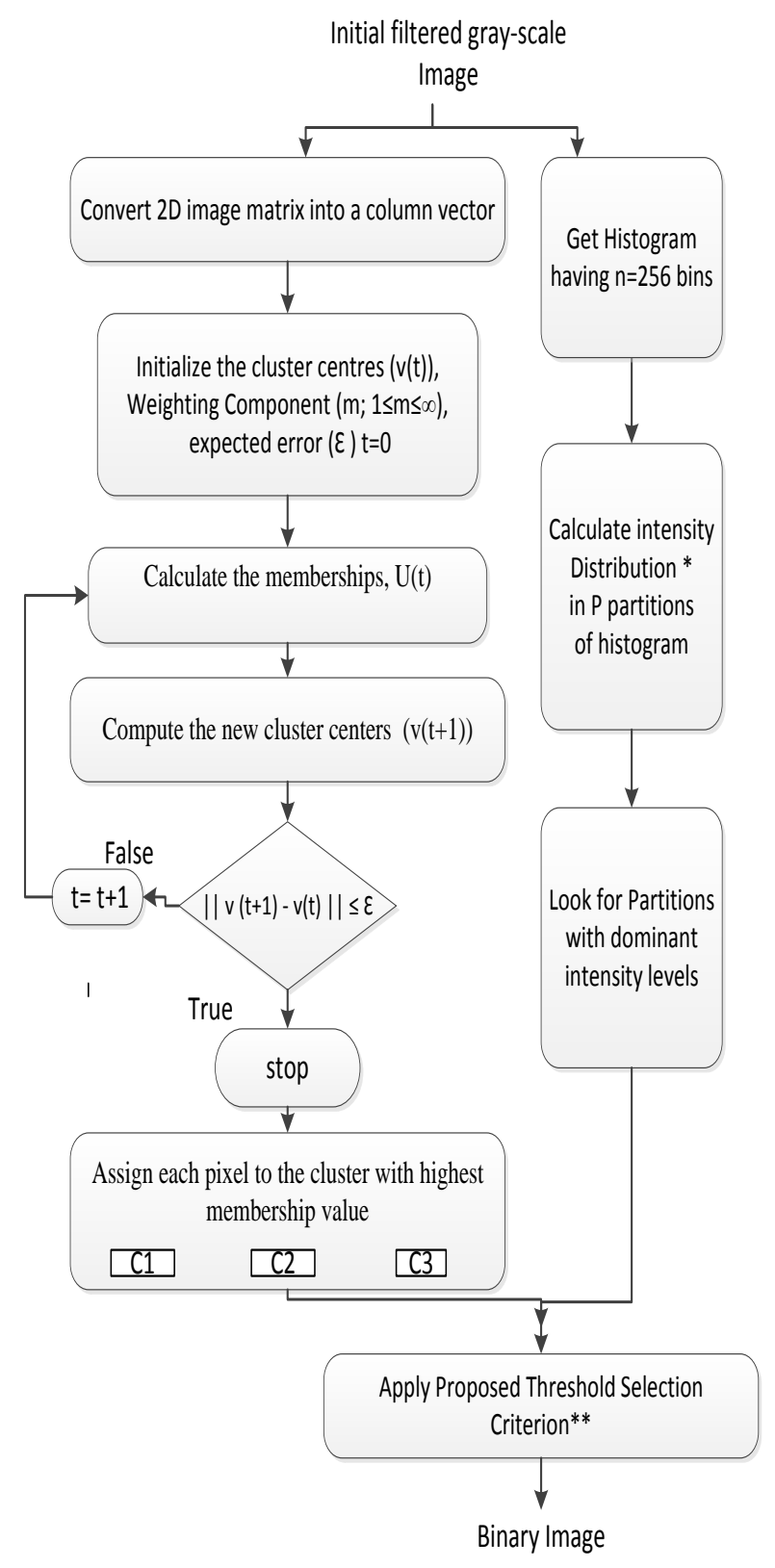

Figure. 4 Histogram Analysis based Fuzzy C Mean Algorithm [15]

Segmentation and thresholding results for some of the skin lesion images are shown in Fig. 5. 

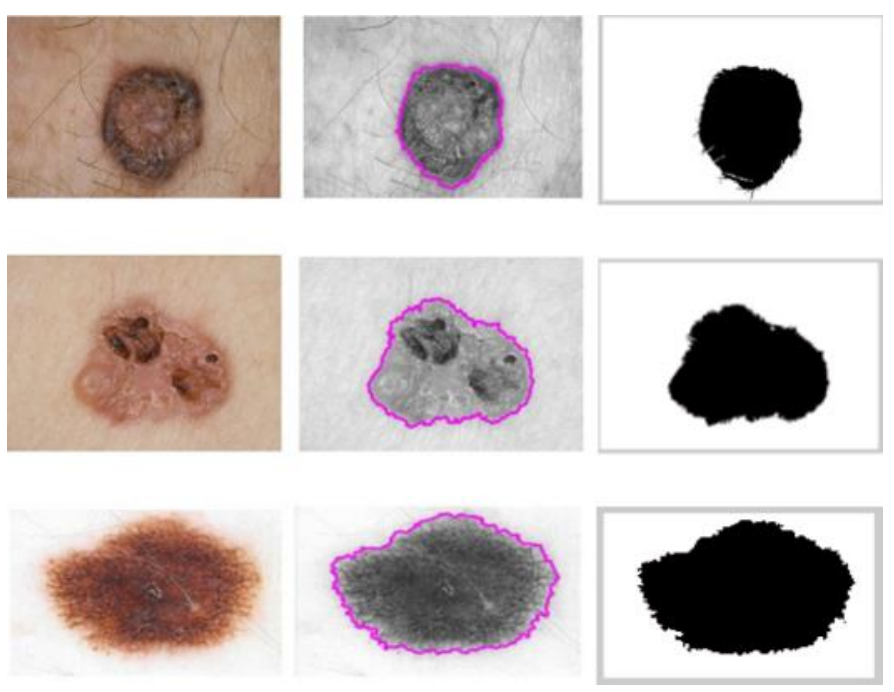

Figure. 5 Segmentation of Skin

\subsection{Feature Extraction}

The features analysis of skin lesions suggest that surface characteristics of the skin lesion changes as it progresses towards melanoma. In the early stages of melanoma the features used in visual examination are hardly visible and thus it may lead to a false diagnosis. However, if images of skin lesions can be collected that has spatial/frequency and texture information, then a non-invasive method of lesion classification based on the surface characteristics can be developed.

When performing analysis of skin lesions it is difficult to obtain a general mathematical model for various textures because of the large variation in their properties. Texture plays a significant role in image analysis and pattern recognition, some studies that implemented on board textural feature extraction can be found in $[17,18]$. In this paper, we used both histogram based features and texturebased features which are consequently used for classification. Histogram based features include,

$$
\begin{aligned}
& \text { Mean } \mu=\sum_{i=1}^{N} p_{i} x_{i} \\
& \text { Variance } \sigma^{2}=\sum_{i=1}^{N} p_{i}\left(x_{i}-\mu\right)^{2} \\
& \text { Standard deviation } \sigma=\sqrt{\sigma^{2}} \\
& \text { Skewness }=\sum_{i=1}^{N} \frac{\left(x_{i}-\mu\right)^{3}}{(N-1)(\sigma)^{2}} \\
& \text { Kurtosis }=\sum_{i=1}^{N} \frac{\left(x_{i}-\mu\right)^{4}}{(N-1)(\sigma)^{4}} \\
& \text { Energy }=\sum_{i=1}^{N}\left(p_{i} \cdot p_{i}\right) \\
& \text { Entropy }=-\sum_{i=1}^{N}\left(p_{i} \cdot \log \left(p_{i}\right)\right)
\end{aligned}
$$

GLCM GLCM (Grey Level Co-occurrence Matrix) introduced by Haralick provides one of the most popular statistical methods in analysis of grey tones in an image [19]. Gray- level co-occurrence matrix (GLCM) is a statistical method for examining the textures that considers the spatial relationship between the pixels [20].
The GLCM features characterize the texture of an image by calculating how often pairs of pixel occur in an image with specific values and in a specified spatial relationship. This calculation is used to create a Gray Level Cooccurrence matrix, and then statistical measures are extracted from this matrix. In GLCM the number of rows and columns is equal to the number of gray levels, $G$, in the image. The matrix element $P(i, j \mid \Delta x, \Delta y)$ is the relative frequency with which two pixels, separated by a pixel distance $(\Delta x, \Delta y)$, occur within a given neighborhood, one with intensity ' $\mathrm{i}$ ' and the other with intensity ' $j$ '. The matrix element $P(i, j \mid d, \theta)$ contains the second order statistical probability values for changes between gray levels ' $i$ ' and ' $j$ ' at a particular displacement distance $\mathrm{d}$ and at a particular angle $(\theta)$. The class mark of $i^{\text {th }}$ intensity level is denoted by $x i$, frequency of $i^{\text {th }}$ level is denoted by fi and the relative frequency of the $\mathrm{i}^{\text {th }}$ level is denoted by

$$
p_{i}=\frac{f_{i}}{N}
$$

The GLCM based features that have been used during feature extraction include

$$
\text { Autocorrelation }=\sum_{i=0}^{N-1} \sum_{j=0}^{N-1}(i x j) p(i, j)
$$

$$
\begin{gathered}
\text { Contrast }=\sum_{n=0}^{N-1} n^{2}\left\{\sum_{i=1}^{N} \sum_{j=1}^{N} p(i, j)\right\},|i-j|=n \\
\text { Correlation }=\sum_{i=0}^{N-1} \sum_{j=0}^{N-1} \frac{(i x j) p(i, j)-\left\{\mu_{x} \times \mu_{y}\right\}}{\sigma_{x} \times \sigma_{y}} \\
\text { Cluster_Prominence }= \\
\sum_{i=0}^{N-1} \sum_{j=0}^{N-1}\left(i+j-\mu_{x}-\mu_{y}\right)^{4} \times p(i, j)
\end{gathered}
$$

Cluster shade $=\sum_{i=0}^{N-1} \sum_{j=0}^{N-1}\left(i+j-\mu_{x}-\mu_{y}\right)^{3} \times p(i, j)$

$$
\text { Dissimilarity }=\sum_{i=0}^{N-1} \sum_{j=0}^{N-1}|i-j| \cdot p(i, j)
$$

$$
\text { Energy }=\sum_{i=0}^{N-1} \sum_{j=0}^{N-1} p(i, j)^{2}
$$

$$
\text { Entropy }=-\sum_{i=0}^{N-1} \sum_{j=0}^{N-1} p(i, j) \times \log (p(i, j))
$$

$$
\begin{aligned}
& \text { Homogeneity }=\frac{\sum_{i=0}^{N-1} \sum_{i=0}^{N-1}(i j) p(i, j)-\mu_{x} \mu_{y}}{\sigma_{x} \sigma_{y}} \\
& \text { Maximum probability= } \operatorname{MAX}_{i, j} p(i, j)
\end{aligned}
$$

Difference variance $=$ variance of $p_{x-y}$

$$
\text { Diff_entropy }=-\sum_{i=0}^{N-1} p_{x-y}(i) \log \left(p_{x-y}(i)\right)
$$

Inverse difference normalized $=\sum_{i, j=1}^{N} \frac{C(i, j)}{1+|i-j|^{2} / N^{2}}$ 


$$
\text { where } C(i, j)=\frac{p(i, j)}{\sum_{i, j=1}^{N} p(i, j)}
$$

Inverse difference moment normalized $=$

$$
\sum_{i, j=1}^{N} \frac{C(i, j)}{1+(i-j)^{2} / N^{2}}
$$

Information measure of correlation $=$ $\frac{-\sum_{i=0}^{N-1} \sum_{j=0}^{N-1} p(i, j) \times \log (p(i, j))+\sum_{i=0}^{N-1} \sum_{j=0}^{N-1} p(i, j) \times \log \left\{p_{x}(i) p_{x}(j)\right]}{\max \left[\text { entropy of } p_{x} \text { entropy of } p_{y}\right\}}$

Information measure of correlation $2=$ $\left(1-\exp \left[-2.0\left(-\sum_{i=0}^{N-1} \sum_{j=0}^{N-1} p(i, j) \times \log \left\{p_{x}(i) p_{x}(j)+\right.\right.\right.\right.$ $\left.\sum_{i=0}^{N-1} \sum_{j=0}^{N-1} p(i, j) \times \log (p(i, j))\right)^{1 / 2}$

\subsection{Classification}

There is a large variety of skin lesions with different features that can indicate cancerous lesions. It is complicated to develop statistical rule based algorithms for diagnosis. Neural network have better capability of handling complex relationships between the different parameters and making classification based on training data [21, 22]. Thus, neural network can perform well for skin lesion diagnostic systems.

In the experimental analysis, a two layer feed forward neural network with the sigmoid activation function is designed with 22 input neurons, 20 hidden neurons and 2 output neurons for the classification. The exact choice of hidden neurons is not possible for various reasons like uncertain and casual nature of learning procedures and also freedom of system under investigation. Thus, the choice of neurons in hidden layer is made empirically through experimental analysis considering best performance accuracy in diagnosis results.

The scaled conjugate gradient algorithm is used for training the neural network. Conjugate gradient algorithms require a line search at each iteration. This line search is computationally expensive, since it requires the network response to all training inputs that are computed several times for each search. The scaled conjugate gradient algorithm (SCG), developed by Moller [23], was designed to avoid the time-consuming line search. This algorithm is too complex to explain in a few lines, but the basic idea is to combine the model-trust region approach used in the Levenberg-Marquardt algorithm with the conjugate gradient approach. A detailed explanation of the algorithm can be found in [23].

\section{Experimental Results}

For training the neural network, 107 images are used, 14 images for validation and 14 images for the testing. The training stops when a classifier gives a higher accuracy value with minimum training and testing errors. Neural Networks is implemented using MATLAB software R2013 and simulated by a system with corei5 $3.10 \mathrm{GHz}$ processor and 4 GB memory under Windows7 operating system.

For evaluating the performance of classification process, the error measures being used in this study include Mean Squared Error (MSE) and Percent Error (PE). MSE is calculated between the desired output and the actual output of the neural network.

The formula use for MSE is as followsn

$$
M S E=\frac{\sum_{j=0}^{P} \sum_{i=0}^{N}\left(d_{i j}-y_{i j}\right)^{2}}{N P}
$$

Where, $\mathrm{P}$ is the number of output processing elements, $\mathrm{N}$ is the number of exemplars in the training data set, $y_{i j}$ is the estimated network output for pattern $i$ at processing element $j$, whereas, $d_{i j}$ is the actual output for exemplar $i$ at processing element $\mathrm{j}$.

Percent Error indicates the percentage of the samples, which are misclassified by the neural network.

$$
\% \text { error }=\frac{100}{N P} \sum_{j=0}^{P} \sum_{i=0}^{N} \frac{\left|d y_{i j}-d d_{i j}\right|}{d d_{i j}}
$$

Where

$\mathrm{P}$ is the number of output processing elements, $\mathrm{N}$ is the number of patterns in the training data set, $\mathrm{dy}_{\mathrm{ij}}$ is the demoralized network output for pattern $i$ at processing element $\mathrm{j}$, whereas, $\mathrm{dd}_{\mathrm{ij}}$ is the demoralized desired network output for exemplar $i$ at processing element $j$.

The confusion matrix, shown in Fig. 6, gives the accuracy of the classification problem. It can be seen that out of 81 benign lesions 74 were correctly classified as non-cancerous and out of 54 melanoma (cancerous) images 50 were correctly diagnosed as cancerous lesions. The overall accuracy (training, testing and validation) of the system is $92 \%$, with the accuracy of test results around $92.9 \%$.

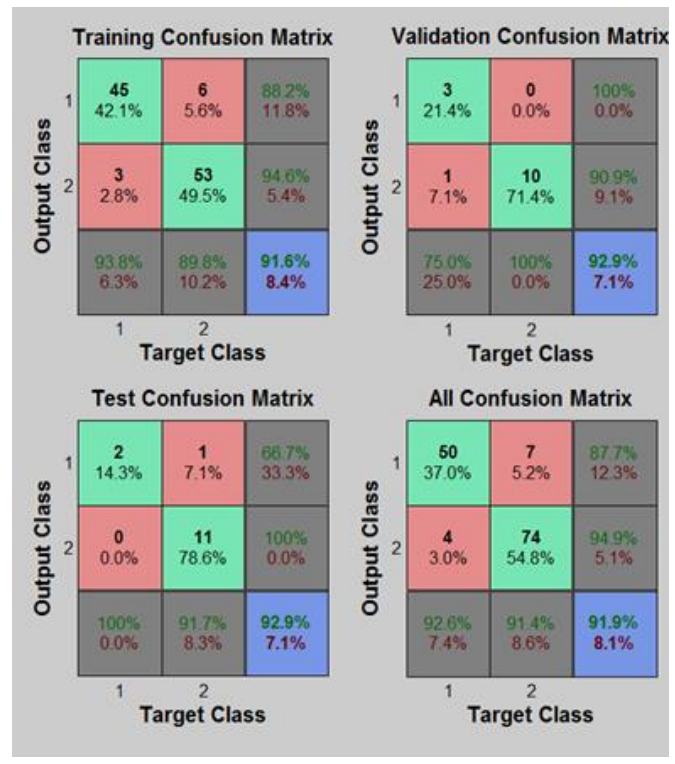

Figure. 6 Confusion Matrix for Classification

Receiver Operating Characteristic curve (or ROC curve.) is a plot of the true positive rate against the false 
positive rate for the different possible cut points of a diagnostic test. It shows the trade-off between sensitivity and specificity (any increase in sensitivity will be accompanied by a decrease in specificity). The closer the curve follows the left-hand border and then the top border of the ROC space, the more accurate the test. The ROC of the proposed diagnostic system shows good diagnostic efficiency of the method as it is shown in Fig. 7.

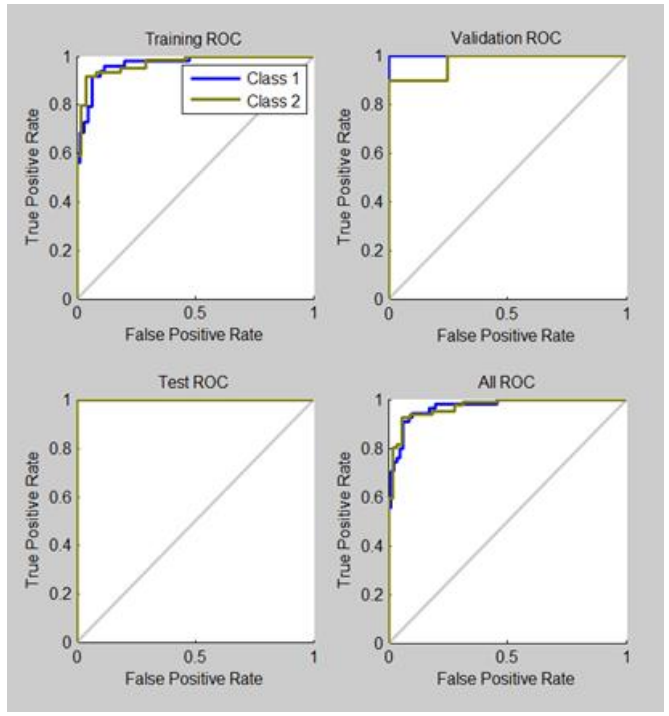

Figure. 7 Receiver Operating Characteristics Curve of Classifier

\subsection{Comparative Analysis}

Performance of scaled conjugate gradient algorithm (SCG) is also compared with some other well-known back propagation neural network learning algorithms like Resilient back propagation (RP) and LevenbergMarquardt (LM) back propagation algorithm. The best performance graphs and respective confusion matrices obtained for the three learning algorithms SCG, LM and $\mathrm{RP}$ is shown in the Fig. 8, 9 and 10 respectively.

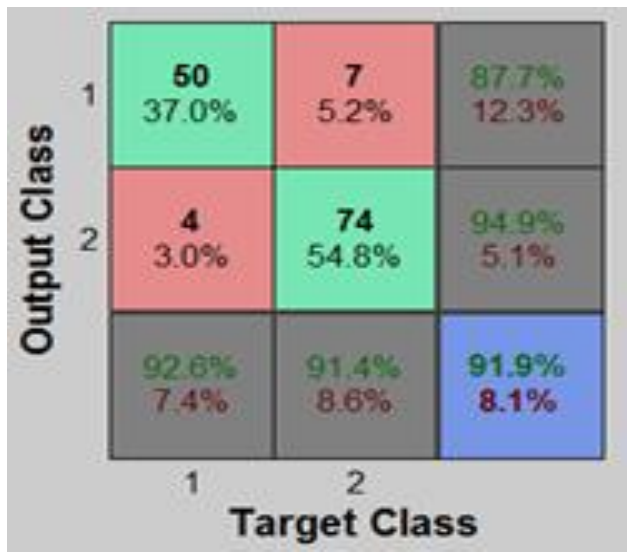

(a)

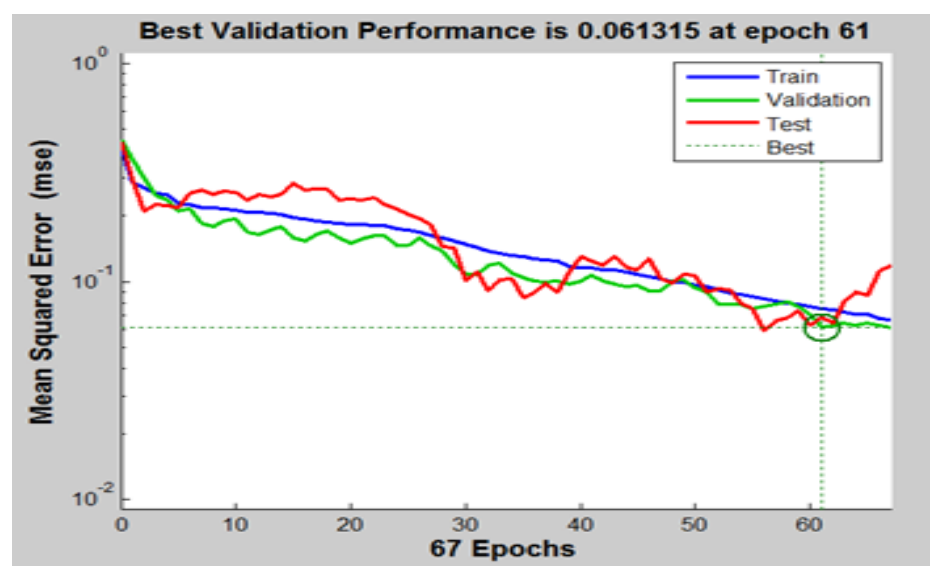

(b)

Figure.8 (a) Confusion Matrix (b) Performance Graph of Scaled Conjugate Gradient Learning Algorithm

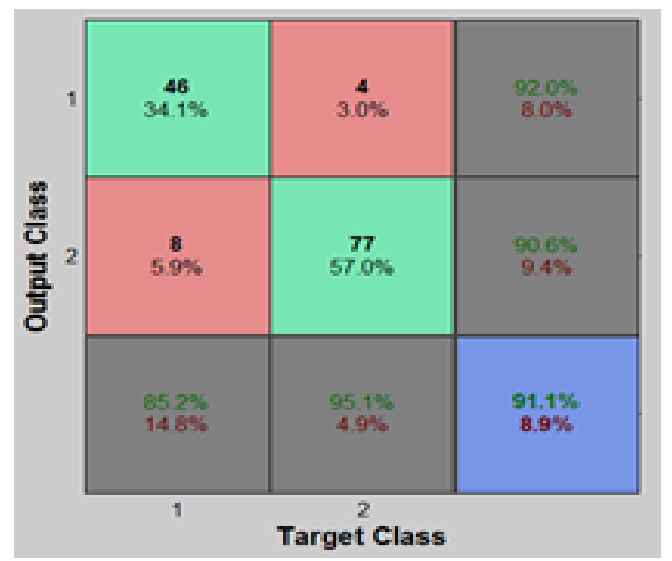

(a)

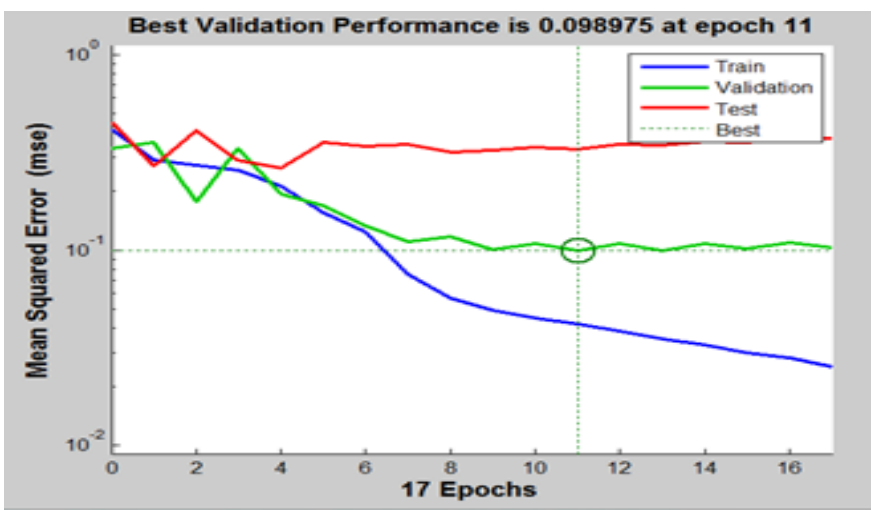

(b)

Figure. 9 (a) Confusion Matirx (b) Performance Graph of Levenberg-Marquardt Learning Algorithm 


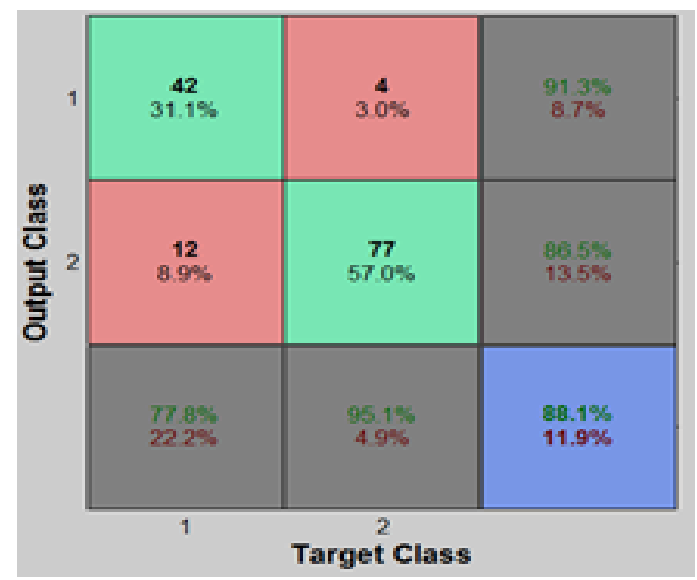

(a)

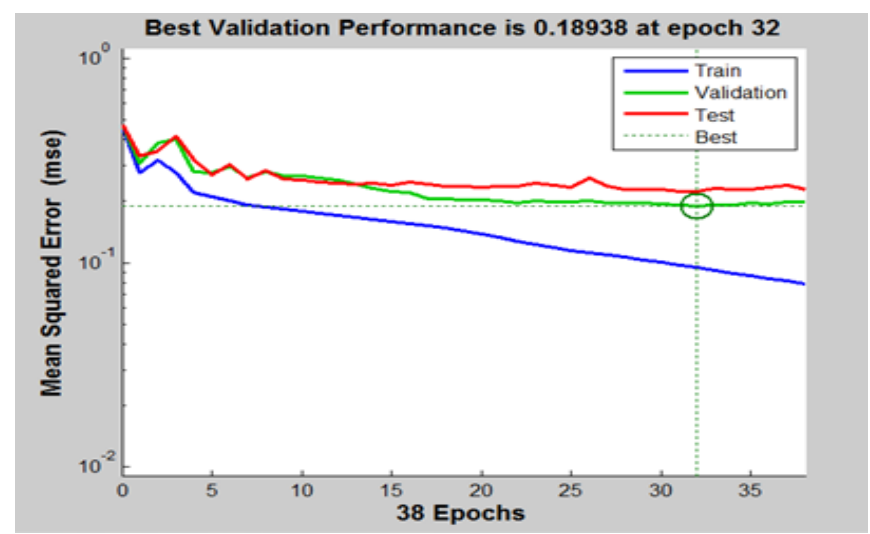

(b)

Figure. 10 (a) Confusion Matrix (b) Performance Graph of Resilient Back Propagation Learning Algorithm

The confusion matrix showing the best diagnosis performance of each training algorithm is presented in figure 8(a), 9(a) and 10(a). It can be seen that the best overall classification $91.9 \%$ was obtained using SCG with sensitivity $92.6 \%$ and specificity $91.4 \%$. The overall performance of LM was close to SCG, but it can be seen that although it worked better in detecting benign lesions specificity $95.1 \%$ but its classification efficiency for melanoma is lower than SCG (sensitivity 85.2\%). While Resilience BP algorithm could acquire accuracy of $88.1 \%$, specificity $95.1 \%$ and sensitivity $77.8 \%$.

For validating the results, hold-out validation is used, where we hold $10 \%$ of the data, selected randomly, for test phase. In order to ensure better validation of the neural network performance the hold-out validation was repeated several times. The overall average performance comparison for the three algorithms is provided in Table 1. It can be observed that SCG training algorithm performed better for overall classification of skin cancer images by classifying the data with minimum performance error and maximum accuracy of $91.1 \%$.
Table 1

Performance Comparison of Learning Algorithms for Neural Network

\begin{tabular}{|c|c|c|c|}
\hline \multicolumn{4}{|c|}{ Training Algorithm } \\
\hline & $\begin{array}{c}\text { Scaled } \\
\text { conjugate } \\
\text { gradient BP }\end{array}$ & $\begin{array}{c}\text { Levenberg- } \\
\text { Marquardt } \\
\text { BP }\end{array}$ & Resilient BP \\
\hline Performance & 0.069 & 0.070 & 0.13 \\
\hline $\begin{array}{c}\text { Training } \\
\text { Performance }\end{array}$ & 0.073 & 0.081 & 0.84 \\
\hline $\begin{array}{c}\text { Validation } \\
\text { Performance }\end{array}$ & 0.08 & 0.094 & 0.12 \\
\hline $\begin{array}{c}\text { Testing } \\
\text { Performance }\end{array}$ & 0.068 & 0.29 & 0.19 \\
\hline $\begin{array}{c}\text { classification } \\
\text { Rate }\end{array}$ & $91.1 \%$ & $89.9 \%$ & $87.5 \%$ \\
\hline
\end{tabular}

\section{Conclusion \& Future Work}

In this study, a methodological approach is presented for the classification of skin lesions. The overall process includes pre-processing, segmentation, feature extraction, and classification based on artificial neural network using scaled conjugate gradient algorithm. The system was tested on a set of 135 images. Despite of the fact that the images came from different sources and there was no control over their acquisition, the result with overall accuracy of $92 \%$ for the present study are significant and seems quite promising for the future research.

This present work is different from earlier studies in several aspects. First of all, the images used in this study came from different sources and acquired using different acquisition systems. For this reason, care was taken to extract features that are invariant with respect to the changes in the imaging conditions. Second, starting from the segmentation until the classification, the whole process was fully automated. Third, for segmentation stage had used a novel algorithm which has proved to outperform other segmentation algorithms used so far for segmentation of skin lesions.

Although a high accuracy is achieved by computer aided diagnostic systems employing statistics obtained from Low-level features such as the one presented in this work. Still we recommend that least two issues need to be addressed before these automated systems gain greater clinical acceptance. First, higher level features based on a particular diagnostic scheme such as pattern analysis, 7 point checklist etc. should be integrated with the existing low level features. It is very likely that more research for 
the extraction of critical features such as atypical pigment networks, globules, and blue-white areas, can increase the diagnostic accuracy of computerized dermoscopy image analysis systems. Second, for developing a more reliable diagnostic system we intend to use multiple classifier based systems to undo the chances of misclassification due to classifier limitations. For this purpose, experiments combining different neural networks and other classification algorithms like support vector machine [24], extreme learning machine [25] etc. would be done

The recent technical achievements in the areas of image acquisition and processing systems can allow the improvement of diagnostic process along with lowering the cost of image analysis systems. Modern technology like the spectral imaging systems can be used to obtain spectral signatures of the tissues as additional features and it can be used for further detailed analysis of lesions. Such tools may serve as diagnostic adjuncts for medical professionals and can be used for having second opinion for the confirmation of a diagnosis. Thus, it will provide us with the opportunity of implementing more accurate, faster and reliable diagnostic systems.

\section{References}

[1] Siegel, R., et al., Cancer statistics, 2011. CA: A Cancer Journal for Clinicians, 2011, vol. 61(4), 212-236.

[2] Society, A.C., Cancer Facts \& Figures 2012, http://www.cancer.org/acs/groups/content/epidemiologysur veilance/documents/document/acspc-031941.pdf 2012.

[3] Causes of death 2010, C.W.o. Australia, Editor, Australian Bureau of Statistics: Canberra, Australia.

[4] Australian, C.C. Cancer Council to launch new research / Failure to monitor highlights cancer risk. 2010; <http://www.cancer.org.au/cancersmartlifestyle/SunSmart/ Skincancerfactsandfigures.htm>.

[5] Sikorski, J. "Identification of malignant melanoma by wavelet analysis", in Student/Faculty Research Day, CSIS, Pace University, 2004.

[6] Ganster, H., et al., Automated melanoma recognition, IEEE Transactions on Medical Imaging, 2001, vol. 20(3), 233 239.

[7] Rubegni, P., et al., Automated diagnosis of pigmented skin Lesions, International Journal of Cancer, 2002, vol.101 (6), 576-580.

[8] Ignazio Stanganelli, A.B., Luigi Calori, Roberto Gori, et al., Computer aided diagnosis of melanocytic tumors, Anticancer Research, 2005, vol. 25, 4577-4582.

[9] Ruiz, D., et al., A decision support system for the diagnosis of melanoma: A comparative approach, Expert Systems with Applications, 2011, vol. 38(12), 15217-15223.

[10] Masood. Ammara., Ali Al-Jumaily, Adel, "Computer Aided Diagnostic Support System for Skin Cancer: A Review of Techniques and Algorithms", International Journal of Biomedical Imaging, p. 22, 2013.

[11] Masood. Ammara, Adel Al Jumaily, "Fuzzy C mean Thresholding based Level Set for Automated Segmentation of Skin Lesions", Journal of Signal and Information Processing, vol. 4(3B), 66-71, July 2013.

[12] Masood, Ammara Adel Al Jumaily, Al Hoshyar, Azadeh
Noori Masood, Omama. "Automated segmentation of skin lesions: Modified Fuzzy C mean thresholding based level set method", Proc. 2013 16th International Multi Topic Conference (INMIC), 201-206, December 2013.

[13] Hwang, H. and R.A. Haddad, Adaptive median filters: new algorithms and results, IEEE Transactions on Image Processing, 1995, vol. 4(4), 499-502.

[14] Sun, T. and Y. Neuvo, Detail-preserving median based filters in image processing, Pattern Recognition Letters, 1994, vol. 15(4), 341-347.

[15] Masood, A.A.-J., Adel Ali. Integrating soft and hard threshold selection algorithms for accurate segmentation of skin lesion, Proc 2014 Middle East Conference on Biomedical Engineering (MECBME), 83-86, 2014.

[16] Ammara Masood, Adel Al Jumaily, Maali, Yashar, "Level Set Initialization Based on Modified Fuzzy C Means Thresholding for Automated Segmentation of Skin Lesions", in Neural Information Processing, Editor M.H. Lee, Akira Hou, Zeng-Guang Kil, RheeMan, Springer Berlin Heidelberg. 341-351, November 2013.

[17] Tanaka, T.T., S. Kabuta, I. Shimizu, K. Tanaka, M. Oka, H. "Pattern classification of nevus with texture analysis", Proc. 26th Annual International Conference of the IEEE Engineering in Medicine and Biology Society, 2004, 14591462.

[18] Parekh, R., "Improving Texture Recognition using Combined GLCM and Wavelet Features", International Journal of Computer Applications, vol. 29(10), 41-46, 2011.

[19] Markus Gipp, G.M., Nathalie Harder, Apichat Suratanee, Karl Rohr, Rainer König, Reinhard Männer, "Haralick’s Texture Features Computed by GPUs for Biological Applications" IAENG International Journal of Computer Science, vol. 36(1), 2009.

[20] Mohanaiah, P.S., L. GuruKumar, "Image Texture Feature Extraction Using GLCM Approach”, International Journal of Scientific and Research Publications (IJSRP), vol. 3(5), $1-5,2013$.

[21] Ho Tak Lau, A.A.-J., "Intelligent Automatically Early Detection of Skin Cancer: Study Based on Neural Network Classification", Proc. International Conference on Soft Computing and Pattern Recognition, 2009, 375-380.

[22] Dreiseitl, S., et al., "A Comparison of Machine Learning Methods for the Diagnosis of Pigmented Skin Lesions", Journal of Biomedical Informatics, 34(1): p. 28-36, 2001.

[23] Moller, M.F., “A scaled conjugate gradient algorithm for fast supervised learning", Neural Networks, vol. 6, 525533, 1993.

[24] Xiaojing, Y., et al., "SVM-based Texture Classification and Application to Early Melanoma Detection”, Proc. 28th Annual International Conference of the IEEE Engineering in Medicine and Biology Society, 2006. New York, USA, 4775-4778.

[25] Huang, G.-B., Q.-Y. Zhu, and C.-K. Siew, "Extreme learning machine: Theory and applications",Neurocomputing, vol. 70(1-3), 489-501, 2006. 